\title{
Análise de preensão dos atletas sub 17 e sub 20 da seleção brasileira de Judô*
}

\author{
Analysis of strength of athletes sub 17 and \\ sub 20 of the Judo Brazilian selection
}

\begin{abstract}
Marcio de Paula Oliveira'
Gabriela Ataides de Oliveira ${ }^{2}$

Jefferson Dias Fernandes ${ }^{3}$

Bruna Cury Lourenço Peres ${ }^{4}$ Juane Juare $z^{5}$
\end{abstract}

* Recebido em: $12 / 09 / 2013$

Aprovado em: 22/05/2014

1 Especialista em Fisioterapia Traumato-Ortopédica Funcional e Esportiva (UCB/RJ); Mestre em Ciências da Saúde (UnB); Doutorando em Medicina Interna e Terapêutica (UNIFESP/ EPM); Fisioterapeuta do Centro de Reabilitação SPORTFISIO, no Hospital Ortopédico e Medicina Especializada - HOME e Chefe do Departamento de Reabilitação do Brasiliense Futebol Clube; Ex-Fisioterapeuta das Categorias de Base da Seleção Brasileira de Judô. Jefferson Dias Fernandes: Bacharel em Fisioterapia (UCB); Especialista em Fisioterapia Traumato-Ortopédica Funcional e Esportiva (Uniceub); Pós-graduando em Docência do Ensino Superior e Profissional; Professor de Anatomia e Fisiologia Humana na Faculdade e Escola Técnica LS. Fisioterapeuta da Clínica de Fisioterapia Multifisio.

2 Fisioterapeuta graduada pelo Centro Universitário de Brasília; Professora de Anatomia Humana na Faculdade e Escola técnica LS.

3 Bacharel em Fisioterapia (UCB); Especialista em Fisioterapia Traumato-Ortopédica Funcional e Esportiva (Uniceub); Pós-graduando em Docência do Ensino Superior e Profissional; Professor de Anatomia e Fisiologia Humana na Faculdade e Escola Técnica LS. Fisioterapeuta da Clínica de Fisioterapia Multifisio.

4 Graduada pelo Centro Universitário de Brasília - UNICEUB; Aprimorada em fisioterapia cardiorespiratória pelo Instituto de Cardiologia do DF; Fisioterapeuta do Instituto de Cardiologia do DF e na Clínica de doenças renais de Brasília.

5 Graduada pelo Centro Universitário de Brasília - UNICEUB.

\section{Resumo}

A força de preensão palmar apresenta um papel importante na execução de tarefas de vida diária do homem e também é bastante utilizada em esportes variados, como, por exemplo, o Judô. Nessa modalidade, a mensuração dessa força toma posição de bastante relevância, influenciando, portanto, no desempenho dos seus atletas. Trata-se de um estudo de caráter analítico transversal, que avaliou a força de preensão palmar em atletas da seleção brasileira de judô sub 17 e sub 20, com objetivo geral de mensurar e analisar a força de preensão palmar dos atletas em estudo e estabelecer um parâmetro de treinamento e reabilitação pós-trauma e pós-cirúrgico dos membros superiores. De uma forma específica, comparou-se a força manual desses atletas (GA) com não praticantes de esporte competitivo (GC) e se verificou se existe correlação entre tempo de treinamento e força de preensão e graduação e força manual. Participaram da pesquisa 89 indivíduos, sendo 60 atletas e 29 não atletas de ambos os gêneros e faixa etária compreendida entre 14 e 19 anos. $\mathrm{O}$ instrumento utilizado para avaliação foi o dinamômetro SAEHAN ${ }^{\circ}$. Com relação à média da força de preensão obtida nos grupos estudados, observou-se que há diferença significativa $(\mathrm{p}<0,05)$ no GA em relação ao GC em ambos os membros, que o tempo de treinamento não influencia de forma significativa no aumento da força de preensão, e observou-se também que existe aumento da força de preensão conforme a graduação do atleta. Os resultados obtidos podem ser considerados em uma reabilitação da função manual do atleta de judô.

Palavras-chave: Judô. Atletas. Força. Preensão Palmar.

\begin{abstract}
The grip strength plays an important role in performing tasks of daily life of man and is also widely used in various sports such as Judo. In this mode, the measurement of that force takes quite relevant position, influencing therefore the performance of their athletes. This is a study of cross sectional nature, which evaluated the grip strength in athletes of the Judo Brazilian selection sub 17 and sub 20, with overall objective of measuring and analyzing the grip strength of the athletes in the study and establish a training and rehabilitation post- trauma and post -surgical parameter of the upper limbs. In a specific way, we compared the strength of these athletes manual (GA) with not practicing competitive sport (GC) and see if there is a correlation between training time and grip strength and hand strength and graduation . 89 individuals participated in the survey, with 60 athletes and 29 non-athletes of both genders and aged from 14 to 19 years. The instrument was used to assess the dynamometer SAEHA. Regarding grip strength obtained in the groups studied average, we observed a significant difference $(\mathrm{p}<0.05)$ in GA than in GC in both members, the training time does not influence significantly the increase grip strength, and it was observed that there is increase in grip strength as undergraduate athlete. The results can be considered in a rehabilitation manual function judo athlete.
\end{abstract}

Keywords: Judo. Athletes. Force. Palmar hold. 


\section{Introdução}

A capacidade da mão em executar tarefas, segurar objetos e imprimir força está relacionada à força de preensão palmar, e, em esportes variados, as mãos são utilizadas em movimentos de alto grau de habilidade, força e resistência muscular, que, juntos, influenciam na obtenção de um bom desempenho (BORGES JUNIOR et al., 2009).

A força se destaca como um fator bastante determinante no rendimento esportivo, e pode ser influenciada por fatores internos como o tipo e número de fibras musculares, secção transversa de fibra muscular, a coordenação e a velocidade e contração dessas fibras, ângulo do movimento, gênero e idade. Existem também fatores externos que interferem no resultado final dessa força, dentre eles o método de treinamento, a motivação, a hora do dia, a nutrição e o doping (FERNANDES; JUNIOR; MARINS, 2005; MCARDLE; KATCH; KATCH, 1992)

Segundo Franchini (2008), um fato não pode ser contestado: o homem luta desde sua origem. Inicialmente, a luta era pela sobrevivência, depois por poder, e, nos dias de hoje, para melhorar o condicionamento físico, diversão, para competir ou evoluir espiritualmente. Barsottini, Guimarães e Morais (2005) relatam que o judô é uma das modalidades esportivas que apresenta elevada adesão na atualidade, principalmente em idades púberes e pré-púberes.

"O judô, que pode ser traduzido como "Caminho Suave", é uma prática esportiva de origem japonesa, sistematizada por Jigoro Kano, em 1882, a partir de antigas técnicas de lutas que ele havia estudado e praticado" (ARAÚJO; ANDRADE; PRADA, 2010). Kano (2008) defendia que uma modalidade esportiva como o judô deveria servir principalmente para educação global dos praticantes, e não como duelo mortal.

Durante a luta de judô, o atleta se encontra em contato com o adversário na maior parte do tempo, e, para manter essa posição, necessita realizar movimentos sucessivos com as mãos mantendo a preensão. Antes da aplicação das técnicas, é aconselhável dominar a pegada e só então o oponente, para que a entrada da técnica e a consequente projeção sejam feitas com maior facilidade e eficácia (FRANCHINI, 2008).

A principal fase em que o posicionamento das mãos determina o domínio do oponente, ou seja, controle do adversário é a de pé. Esse controle é realizado aplicar técnicas do judô, $\mathrm{o}$ atleta realiza preensão palmar, necessitando de uma força muscular adequada para executar o movimento especifico e gerar vantagem durante a luta (KANO, 2008). Apesar da importância dessa força na prática desse esporte, poucos são os estudos que analisam esse aspecto em seus atletas.

Segundo Ruiz-Ruiz et al. (2002), Oliveira et al. (2002) e Moreira et al. (2003), a medição da força de preensão palmar com dinamômetro manual é um teste simples e econômico, capaz de fornecer informações práticas sobre o músculo, nervo, osso ou distúrbios da articulação. Da mesma forma, esses valores representam um bom índice para o processo de reabilitação em lesões que afetam as extremidades superiores, e, nesse caso, serão de notável relevância na reabilitação de atletas do judô. Os dados colhidos auxiliarão o médico e o terapeuta a interpretar resultados e a estabelecer metas adequadas de tratamento, além da aplicação clínica de avaliação da inabilidade, resposta ao tratamento e avaliação da habilidade de um paciente em retornar às atividades de vida diária (MOURA; MOREIRA; CAIXETA, 2008).

Considerando a importância da força de preensão palmar para os atletas de judô, o presente trabalho teve por objetivo geral quantificar e analisar essa força em atletas das categorias Sub17 e Sub20 da Seleção Brasileira de Judô a fim de oferecer dados para servir como parâmetro para treinamento e também para reabilitação após trauma e cirurgia de membros superiores em atletas dessa modalidade e faixa etária.

\section{Materiais e métodos}

Foi realizado um estudo transversal, no qual foi analisada a força de preensão palmar de 60 atletas, $28 \mathrm{mu}$ lheres e 32 homens, das categorias Sub 17 (14 a 16 anos) e Sub 20 (17 a 19 anos) da Seleção Brasileira de judô, e de 29 indivíduos não atletas, 13 mulheres e 16 homens, formando o grupo controle.

Para a obtenção dos dados dos atletas, foram utilizados os prontuários da avaliação fisioterapêutica pré-participação, realizada durante os campeonatos Sul-Americano e Pan-Americano de 2010, que ocorreram respectivamente nos meses de julho e setembro, na Argentina e nos Estados Unidos.

Como critério de inclusão, esses atletas não poderiam apresentar, no momento da avaliação, qualquer história recente de lesões que dificultassem ou incapacitassem a realização do procedimento. Atletas que, mesmo 
tendo sido avaliados, foram excluídos da delegação por lesão, inadequação ao peso da categoria e/ou outros motivos também não participaram da amostra.

\section{Avaliação}

A avaliação foi realizada por um único fisioterapeuta membro da Comissão Técnica da Seleção Brasileira de Judô, e o instrumento utilizado para a avaliação da força de preensão foi o dinamômetro hidráulico SAEHAN ${ }^{\circledR}$, com a manopla ajustada na $2^{\mathrm{a}}$ posição:

Figura 1 - Dinamômetro hidráulico SAEHAN ${ }^{\circledR}$

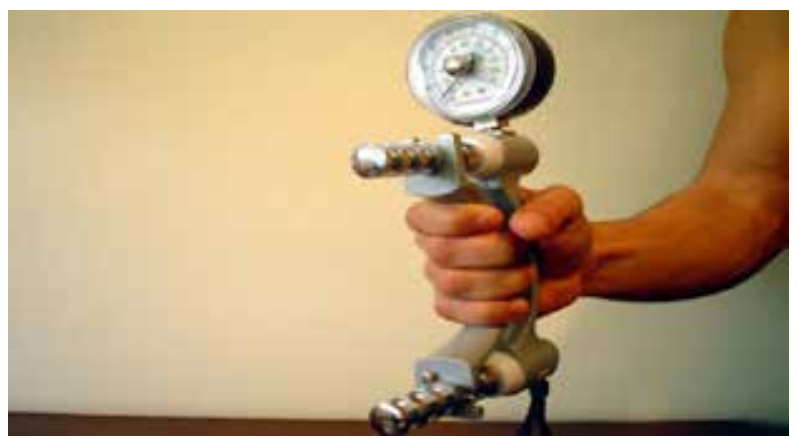

Fonte: foto por Márcio de Paula Oliveira

Durante a realização dos exames, os atletas foram orientados a se manterem sentados de tal maneira que os quadris e joelhos permanecessem a $90^{\circ}$, com os pés apoiados no chão. Para isso, eles foram posicionados em diferentes superfícies, que variaram desde cadeiras a arquibancada, ajustando o posicionamento correto à estatura da pessoa avaliada.

Com relação ao posicionamento do membro superior, tem-se que o ombro se manteve em posição aduzida junto ao tronco, o cotovelo a $90^{\circ} \mathrm{com}$ o antebraço em posição neutra (entre a pronação e supinação) e o punho na posição neutra sem que houvesse desvios, enquanto o examinador sustentava o dinamômetro, seguindo a recomendação da Sociedade Americana de Terapeutas de Mão - SATM (Figura 2). Os sujeitos foram orientados a realizar o movimento de preensão para cada tentativa após o comando verbal do examinador, que consistiu na pronúncia da seguinte frase: "um, dois, três e já". Foram realizadas três repetições, alternando a movimentação para o teste, sendo inicialmente testada a mão direita e logo em seguida a mão esquerda, seguindo criteriosamente a instrumentação do aparelho. O intervalo de tempo entre uma tentativa e outra foi de 30 segundos a fim de que não houvesse fadiga muscular durante o teste. A força foi aplicada durante 5 segundos para cada medida.
Figura 2 - Procedimento de Coleta dos Dados do GA

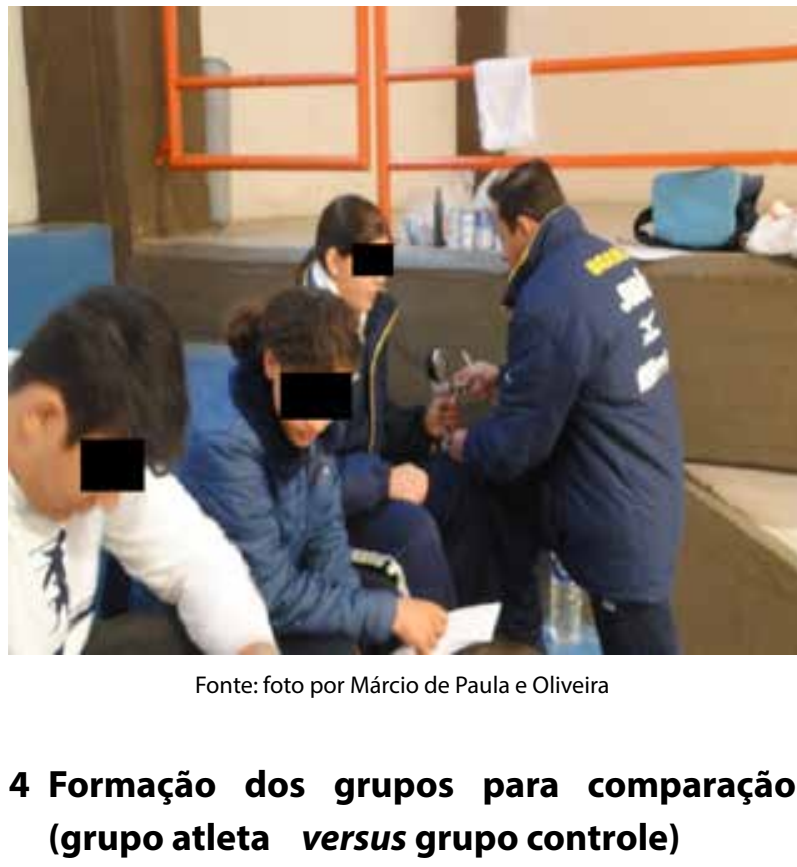

Para comparação dos valores entre atletas e não atletas de modo a verificar se há diferença significativa nos valores de força de preensão palmar, foi formado, por conveniência, um grupo controle GC, por aproximação ao primeiro grupo em gênero, peso e faixa etária, porém formado por indivíduos que não praticavam exercício físico regular e em nível competitivo, não apresentassem lesões em membro superior ou qualquer outro motivo que incapacitasse a realização do procedimento. Estes foram avaliados de acordo com a metodologia utilizada no GA, pelos pesquisadores do estudo. Todos foram voluntários e assinaram um Termo de Consentimento Livre e Esclarecido, dando ciência de que conheciam os objetivos de pesquisa.

A utilização das informações citadas e o desenvolvimento do presente estudo foram autorizados, mediante a assinatura de um Termo de Ciência Institucional, pelo Coordenador Técnico das Categorias de Base da Confederação Brasileira de Judô, e do Comitê de Ética e Pesquisa (CAAE 0166/10). Todos os participantes concordaram em participar da pesquisa e assinaram o Termo de Consentimento Livre e Esclarecido (TCLE).

\section{Análise dos dados}

Para analisar os dados, foi realizada inicialmente a análise descritiva, com a finalidade de definir um panorama geral dos atletas dos grupos Sul-Americano (GSL) e Pan-Americano (GPA) a partir das suas características gerais: identificação, gênero, idade, categoria, tempo de 
treinamento e graduação.

A análise da força de preensão foi realizada com base na metodologia abaixo:

Calculou-se a média e o desvio padrão da preensão palmar da mão direita e da mão esquerda segundo as categorias de peso e gênero. Para avaliar a normalidade das variáveis, utilizou-se o teste de Shapiro-Wilk, logo foi feita a análise de variância não paramétrica (teste de Kruskal-Wallis) para checar se havia diferenças significativas entre a preensão palmar (mão direita e esquerda) entre grupos de atletas e não atletas (controle). Para verificar se existe correlação entre o tempo de treinamento e o aumento da força de preensão, ajustou-se um modelo de regressão linear e para a análise diferencial do aumento da força de preensão palmar foi realizado o teste de comparação múltipla de Kruskal-Wallis.

Por último, os dados foram comparados com um grupo controle, e obtida a diferença numérica, percentual e análise da variância.
O nível de significância estatística adotado nesse trabalho foi de $5 \%$. As análises foram feitas no programa de linguagem estatística $R$.

\section{Resultados}

A amostra foi composta por 60 atletas, sendo que 2 atletas do Pan- americano foram excluídos ao se apresentarem a delegação, 1 feminino por sobrepeso e 1 masculino por lesão. Para manter o pareamento das categorias, foram excluídos 2 atletas do campeonato Sul- americano ( 1 feminino e um masculino) do peso respectivo.

Segundo os objetivos apresentados nesse estudo, os resultados das tabelas 1 e 2 são referentes à força de preensão palmar dos atletas, divididas em categoria e gênero de ambos os membros superiores, estabelecendo uma escala de funcionalidade para servir como parâmetro de reabilitação após traumas ou cirurgia.

As médias e o desvio padrão da força de preensão palmar foram calculados, entre atletas da mesma categoria e peso dos diferentes campeonatos.

Tabela 1- Preensão palmar média na categoria feminino juvenil e feminino júnior

\begin{tabular}{|c|c|c|c|c|c|c|c|c|}
\hline \multirow{2}{*}{ Peso (Kg) } & \multicolumn{2}{|c|}{ Mão Direita juvenil } & \multicolumn{2}{|c|}{ Mão Esquerda juvenil } & \multicolumn{2}{|c|}{ Peso } & \multicolumn{2}{c|}{ Mão Direita júnior } \\
\hline & Média & Desvio Padrão & Média & Desvio Padrão & $\mathbf{( K g )}$ & Média & Desvio Padrão & Média \\
\hline$<40$ & 25,67 & 2,35 & 24,34 & 3,3 & $<44$ & 35,67 & 0,94 & 30,17 \\
\hline$<44$ & 29,5 & 6,36 & 29,84 & 4,01 & $<48$ & 30,5 & 1,17 & 26,83 \\
\hline$<48$ & 28,67 & 1,89 & 25,67 & 3,77 & $<57$ & 41,17 & 3,54 & 36,34 \\
\hline$<52$ & 37,5 & 7,31 & 35,17 & 0,23 & $<63$ & 35,84 & 7,3 & 37,67 \\
\hline$<63$ & 38,84 & 1,65 & 36,67 & 1,41 & $<70$ & 42,67 & 2,35 & 41,17 \\
\hline$<70$ & 40,34 & 10,37 & 36,17 & 9,19 & $<78$ & 39,17 & 4,48 & 37,34 \\
\hline$>70^{*}$ & 33 & 5,19 & 42,5 & 3,07 & & & \\
\hline
\end{tabular}

* Categoria de peso livre.

Tabela 2 - Preensão palmar média na categoria masculino júnior e juvenil

\begin{tabular}{|c|c|c|c|c|c|c|c|c|c|}
\hline \multirow{2}{*}{ Peso (Kg) } & \multicolumn{2}{|c|}{ Mão Direita } & \multicolumn{2}{|c|}{ Mão Esquerda } & \multirow{2}{*}{$\begin{array}{l}\text { Peso } \\
(\mathrm{Kg})\end{array}$} & \multicolumn{2}{|c|}{ Mão Direita } & \multicolumn{2}{|c|}{ Mão Esquerda } \\
\hline & Média & Desvio Padrão & Média & Desvio Padrão & & Média & Desvio Padrão & Média & Desvio Padrão \\
\hline$<55$ & 43,34 & 0,94 & 40,67 & 4,72 & $<50$ & 45 & 0,47 & 49,17 & 8,72 \\
\hline$<60$ & 45,33 & 5,66 & 36,67 & 3,77 & $<55$ & 40 & 3,78 & 38,67 & 8,96 \\
\hline$<66$ & 59,5 & 2,59 & 58,84 & 8,72 & $<60$ & 39,5 & 9,19 & 39,67 & 4,24 \\
\hline$<73$ & 54,67 & 7,54 & 55,5 & 8,24 & $<66$ & 49,67 & 11,31 & 51,17 & 4,95 \\
\hline$<81$ & 57,83 & 7,78 & 50 & 4,71 & $<73$ & 51,67 & 8,96 & 52 & 11,31 \\
\hline$<90$ & 53,34 & 3,77 & 48,34 & 9,43 & $<81$ & 63,34 & 3,77 & 58 & 9,43 \\
\hline$<100$ & 68,34 & 13,67 & 63,5 & 21,45 & $<90$ & 71,83 & 9,19 & 67 & 13,68 \\
\hline$>100^{*}$ & 58 & 7,54 & 56,5 & 10,14 & & & & & \\
\hline
\end{tabular}


De acordo com o teste de Kruskal-Wallis, ao nível de $5 \%$ de significância estatística, em todas as categorias a média da preensão palmar de ambas as mãos dos atletas é superior em relação à preensão palmar de não atletas. As respectivas diferenças e p-valor podem ser observadas na Tabela 3 e 4.

Tabela 3 - Mediana, média \pm desvio padrão para a preensão palmar da mão direita, diferenças entre o grupo de atletas e grupo controle e respectivos p-valores segundo a Anova não-paramétrica -Kruskall-Wallis.

\begin{tabular}{|c|c|c|c|c|}
\hline Grupos & Mediana & Média \pm Desvio & Diferença atleta em relação a controle & p-valor* \\
\hline \multicolumn{5}{|c|}{ Masculino Juvenil } \\
\hline Atleta & 45,66 & $51,57 \pm 12,88$ & \multirow{2}{*}{$23,19 \%$} & 0,0206 \\
\hline Controle & 38,33 & $39,61 \pm 4,89$ & & \\
\hline \multicolumn{5}{|c|}{ Masculino Júnior } \\
\hline Atleta & 54,33 & $55,04 \pm 9,34$ & \multirow{2}{*}{$22,69 \%$} & \multirow{2}{*}{0,0015} \\
\hline Controle & 41,00 & $42,55 \pm 6,44$ & & \\
\hline \multicolumn{5}{|c|}{ Feminino Juvenil } \\
\hline Atleta & 32,66 & $33,36 \pm 6,93$ & \multirow{2}{*}{$25,78 \%$} & \multirow{2}{*}{0,0051} \\
\hline Controle & 24,33 & $24,76 \pm 3,11$ & & \\
\hline \multicolumn{5}{|c|}{ Feminino Júnior } \\
\hline Atleta & 37,5 & $37,5 \pm 5,13$ & \multirow{2}{*}{$26,67 \%$} & \multirow{2}{*}{0,0048} \\
\hline Controle & 29,33 & $27,5 \pm 4,96$ & & \\
\hline
\end{tabular}

Tabela 4 - Mediana, média \pm desvio padrão para a preensão palmar da mão esquerda, diferenças entre o grupo de atletas e grupo controle e respectivos $p$-valores segundo a Anova não- paramétrica - Kruskall-Wallis.

\begin{tabular}{|c|c|c|c|c|}
\hline Grupos & Mediana & Média \pm Desvio & Diferença atleta em relação a controle & p-valor \\
\hline \multicolumn{5}{|c|}{ Masculino Juvenil } \\
\hline Atleta & 49,50 & $50,81 \pm 11,71$ & \multirow{2}{*}{$24,66 \%$} & 0,0046 \\
\hline Controle & 39,61 & $38,28 \pm 3,69$ & & \\
\hline \multicolumn{5}{|c|}{ Masculino Júnior } \\
\hline Atleta & 49,5 & $51,25 \pm 11,63$ & \multirow{2}{*}{$21,31 \%$} & \multirow{2}{*}{0,0234} \\
\hline Controle & 41,67 & $40,33 \pm 8,57$ & & \\
\hline \multicolumn{5}{|c|}{ Feminino Juvenil } \\
\hline Atleta & 33,83 & $32,90 \pm 7,08$ & \multirow{2}{*}{$29,94 \%$} & \multirow{2}{*}{0,004} \\
\hline Controle & 22,67 & $23,05 \pm 4,22$ & & \\
\hline \multicolumn{5}{|c|}{ Feminino Júnior } \\
\hline Atleta & 34,33 & $34,91 \pm 5,78$ & \multirow{2}{*}{$25,35 \%$} & \multirow{2}{*}{0,0087} \\
\hline Controle & 27,17 & $26,06 \pm 4,32$ & & \\
\hline
\end{tabular}

Associando a força de preensão palmar com os anos de treinamento, tentou-se ajustar uma reta que pudesse explicar uma possível relação. Com uma explicação de 6\%, demonstrou-se um ajuste ruim, indicando uma relação fraca, havendo necessidade de outras variáveis para poder definir melhor tal relação.

Segundo o teste de comparações múltiplas de Kruskal-Wallis, há diferenças significativas na preensão palmar média da mão direita em relação às graduações (p-valor 0,0013). Há mudanças significativas na preensão palmar da faixa verde para preta. Também há mudanças da faixa roxa para marrom e preta. Da faixa verde para a roxa e marrom não há mudanças, nem da marrom para a preta. A Tabela 5 apresenta esse comportamento.

Também se notou mudanças significativas na preensão palmar média da mão esquerda em relação às graduações ( $\mathrm{p}$-valor 0,0068). Somente há mudanças significativas na preensão palmar da faixa roxa para a marrom e preta. A Tabela 7 ilustra esse resultado. 
Tabela 5 - Preensão palmar mediana média \pm desvio padrão para a mão direita e esquerda segundo a graduação dos atletas**.

\begin{tabular}{|c|c|c|c|c|}
\hline Faixa & \multicolumn{2}{|c|}{ Mão Direita } & \multicolumn{2}{c|}{ Mão Esquerda } \\
\hline & Mediana & Média \pm Desvio & Mediana & Média \pm Desvio \\
\hline Verde & 36,33 & $36,33 \pm 0,47 \mathrm{bc}$ & 39.17 & $39,17 \pm 7,78 \mathrm{~b}$ \\
\hline Roxa & 31,33 & $31,09 \pm 4,28 \mathrm{c}$ & 29.33 & $31,00 \pm 5,23 \mathrm{ab}$ \\
\hline Marrom & 44,33 & $46,89 \pm 12,01 \mathrm{ab}$ & 42.67 & $44,24 \pm 11,89 \mathrm{a}$ \\
\hline Preta & 49,33 & $51,27 \pm 12,85 \mathrm{a}$ & 46.67 & $49,58 \pm 13,94 \mathrm{a}$ \\
\hline
\end{tabular}

**letras iguais na coluna indicam que as faixas não diferem significativamente, ao nível de 5\% de significância estatística, segundo o teste de comparações múltiplas de Kruskal-Wallis.

\section{Discussão}

A força de preensão palmar está relacionada a atividades da vida diária (AVD). Exceto para locomoção, usamos a mão para quase todas as tarefas em casa, no trabalho e lazer. A diminuição dessa força acaba gerando uma incapacidade física bastante significativa para o indivíduo. Portanto, a força de preensão palmar não é simplesmente uma medida de força da mão ou limitada à avaliação do membro superior, ela tem muitas aplicações clínicas diferentes. Por exemplo, é usada como indicador da força total do corpo, e, nesse sentido, é empregada em testes de aptidão física ou incapacidade de trabalho (QUEIROZ, 2006).

Segundo Barsottini et al. (2006), o judô apresenta um elevado risco para ocorrência de lesões. Ele é apontado com destacado risco relativo quando comparado com outras modalidades esportivas e apresenta ainda uma elevada prevalência para ocorrência de lesões em dedos de mãos e pés. Levando em consideração essa informação, nota-se a relevância para obtenção de valores de preensão manual como parâmetro na reabilitação de lesões em mãos desses atletas.

Muitas vezes nos deparamos com a dificuldade de avaliar o resultado de tratamento de doença traumática de membro superior através da força de preensão palmar, pois não temos o resultado prévio da medida da força (CAPORRINO et al., 1998). Borges Junior et al. (2009) ressalta a importância de se desenvolver protocolos específicos para o fortalecimento das mãos, para assim prevenir lesões nas diversas modalidades esportivas em que o movimento de preensão é utilizado. Sendo assim, conforme o objetivo geral do estudo obteve-se dados dos atletas da seleção brasileira de judô sub 17 e sub 20, para serem utilizados como parâmetro em uma futura reabilitação de traumas ou cirurgia.

Os resultados obtidos no presente estudo indicam que a prática do judô leva a um aumento de força mus- cular para o movimento de preensão palmar, tendo uma diferença significativa em relação a não praticantes. Essa diferença pode ser em decorrência de o tipo de preensão adotado na luta de judô exigir maior manutenção de força durante o combate, implicando maior desenvolvimento dessa variável no grupo de judocas (FRANCHINI; TAKITO; PEREIRA, 2010). Resultados em consenso com Fernandes, Júnior e Marins (2005), que afirmam que os níveis de força manual são influenciados significativamente pela especificidade do movimento de preensão na modalidade esportiva, bem como o tipo ou técnica de treinamento. Oliveira et al. (2006), que em seu estudo analisaram a força de preensão palmar em atletas de jiu-jitsu com relação à graduação e ao tempo de treinamento, mostraram não existir uma relação entre estes parâmetros e o aumento de força de preensão, e que, embora se suponha que ambos sejam diretamente relacionados, nem sempre o atleta mais graduado treina há mais tempo. Isso se deve ao fato de que o atleta que compete bem ou demonstra uma aptidão natural à luta é graduado mais rapidamente, sendo consideradas variáveis distintas, no presente estudo encontraram-se diferentes resultados, ou seja, o tempo de treinamento não influi de forma significativa na força de preensão dos atletas de judô, ao contrário da graduação, que apresentou uma diferença relevante.

No decorrer do estudo, verificamos, em diferentes literaturas, a presença de outras variáveis que influenciam na força de preensão palmar, que, conforme Romero et al. ${ }^{15}$, incluem a idade, gênero, diferentes ângulos do ombro, cotovelo, antebraço, punho e postura. Já Boadella et al. (2005), afirma que a mensuração da força de preensão máxima é um teste simples e barato, mas a eficiência do protocolo é muitas vezes dificultada pela sua complexidade em termo das diferentes posições de preensão, o número de tentativas durante os testes, e a postura corporal na qual o teste foi executado. Observa-se a importância de citá-las para serem analisadas em futuros estudos. 


\section{Conclusão}

Com base nos resultados obtidos neste trabalho, pode-se concluir que os atletas de judô deste estudo apresentam valores elevados de força de preensão palmar, sendo verificados valores significativamente maiores que a população de não atletas tanto para a mão direita quanto para a esquerda; o tempo de prática do esporte não influenciou de forma significativa no aumento da força de preensão palmar dos nossos atletas; conforme a evolução da graduação do atleta houve o aumento da força de preensão palmar. Portanto, esses resultados podem servir como referência de força para a reabilitação da função manual do atleta de judô, bem como também podem servir como referencial para o seu treinamento, auxiliando a equipe técnica e a equipe responsável pela sua reabilitação.

\section{Referências}

ARAÚJO, R. A.; ANDRADE, L. R. Q.; PRADA, F. J. A. A incidência de lesões no joelho em atletas de judô. Revista Digital, Buenos Aires, v. 14, n. 134, jul. 2009.

BARSOTTINI, D.; GUIMARÃES, A. E.; MORAIS P. R. Relação entre técnicas e lesões em praticantes de judô. Revista Brasileira de Medicina do Esporte, São Paulo, v. 12 , n. 01 , p. 56-60, jan./fev. 2006. doi: 10.1590/S151786922006000100011

BOADELLA, M. J.; KUIJER, P. P.; SLUTIER, J. K. Effect of self-selected handgrip position on maximal handgrip strength. Archives of Physical Medicine and Rehabilitation, Chicago, v. 86 , n. 2, p. 328-331, Feb. 2005. doi: 10.1016/j.apmr.2004.05.003

BORGES JUNIOR, N. G. et al. Estudo comparativo da força de preensão isométrica máxima em diferentes modalidades esportivas. Revista Brasileira de Cineantropometria \& Desempenho Humano, Florianópolis, v. 11, n. 3, p. 292-298, jul./set. 2009.

CAPORRINO, F.A. et al. Estudo populacional da força de preensão palmar com dinamômetro Jamar. Revista Brasileira de Ortopedia, Rio de Janeiro, v. 33, n. 2, p.150154, fev. 1998
FERNANDES, A.; JUNIOR, R. A.; MARINS, J. C. B. Estudo comparativo da força de preensão manual entre diferentes modalidades esportivas. Coleção Pesquisa em Educação Física, São Paulo, v. 4, p. 37-39, jun. 2005.

FRANCHINI E.; TAKITO, M. Y.; PEREIRA, J. N. Frequência cardíaca e força de preensão manual durante a luta de jiu-jitsu. Revista Digital, Buenos Aires, v. 9, n. 65, oct. 2003.

FRANCHINI, E. Judô. São Paulo: Odysseus, 2008.

KANO, J. Energia mental e física: escritos do fundador do Judô. São Paulo: Pensamento, 2008.

MCARDLE, W.D.; KATCH, F.I; KATCH, V.L. Fisiologia do exercício: energia, nutrição e desempenho humano. 3. ed. Rio de Janeiro: Guanabara Koogan, 1992.

MOREIRA, D.; ÁlVAREZ, R. R. A.; GODOY, J. R. Abordagem sobre preensão palmar utilizando o dinamômetro $\mathrm{JAMAR}^{\circledR}$ : uma revisão de literatura. Revista Brasileira de Ciência e Movimento, Taguatinga, v. 11, n. 2, p. 95-99, abr./jun. 2003.

MOURA, P. M.; MOREIRA, D.; CAIXETA, A. P. L. Força de preensão palmar em crianças e adolescentes saudáveis. Revista Paulista Pediatria, São Paulo, v. 26, n. 3, p. 290 294, set. 2008. doi: 10.1590/S0103-05822008000300014

OLIVEIRA, M.; MOREIRA, D.; GODOY, J.R.P. Avaliação da força de preensão palmar em atletas de jiu-jitsu de nível competitivo. Revista Brasileira de Ciência e Movimento, Taguatinga, v. 14, n. 3, p. 63-70, jul./set. 2006.

QUEIROZ, J. C. F. Correlação entre a força de pressão palmar e a força da musculatura respiratória em indivíduos obesos e não-obesos. 55f. 2006. Dissertação (Mestrado)- Programa de Pós-Graduação em Educação Física da Universidade Católica de Brasília, Brasília, 2006.

ROMERO, V. E. et al. Hand span influences optimal grip span in boys and girls aged 6 to 12 years. The Journal of Hand Surgery, St. Louis, v. 33, n. 3, p. 378-384, mar. 2008. doi: 10.1016/j.jhsa.2007.11.013

RUIZ-RUIZ, J.; MESA, J. L. M; GUTIÉRREZ, A. Hand size influences optimal grip span in women but not in men. The Journal of Hand Surgery, St. Louis, v. 27, n. 5, p. 897-901, Sep. 2002. doi: 10.1053/jhsu.2002.34315 